\title{
TOPOLOGICAL DUALS OF SOME PARANORMED SEQUENCE SPACES
}

\author{
NANDITA RATH
}

Received 1 October 2002

\begin{abstract}
Let $P=\left(p_{k}\right)$ be a bounded positive sequence and let $A=\left(a_{n k}\right)$ be an infinite matrix with all $a_{n k} \geq 0$. For normed spaces $E$ and $E_{k}$, the matrix $A$ generates the paranormed sequence spaces $[A, P]_{\infty}\left(\left(E_{k}\right)\right),[A, P]_{0}\left(\left(E_{k}\right)\right)$, and $[A, P]((E))$, which generalise almost all the well-known sequence spaces such as $c_{0}, c, l_{p}, l_{\infty}$, and $w_{p}$. In this paper, topological duals of these paranormed sequence spaces are constructed and general representation formulae for their bounded linear functionals are obtained in some special cases of matrix $A$.
\end{abstract}

2000 Mathematics Subject Classification: 40A05, 40C05, 40H05, 46A35, 46A45.

1. Introduction. In the last few decades, Köthe [3], Maddox [4, 5, 6], Simons [11], and several others have made a significant contribution to the study of topological duals of real- and complex-valued sequence spaces. One of the classic problems in the study of topological duals of paranormed sequence spaces is to obtain representation formulae for the elements in their dual spaces. A decisive break with the classical approach is made in this paper by introducing vector-valued sequence spaces in place of sequences of numbers. Here, we study the topological duals of vector-valued sequences which are generated by infinite matrices.

The topological dual of a normed linear space is the set of all continuous linear functionals on the space. Such duals of classical sequence spaces like $c_{0}, c, l_{p}$, and $w_{p}$ [7] are well-known sequence spaces, while the dual of $l_{\infty}$ is a function space. But in the case of a sequence space which is generated by an infinite matrix, the topology is most often induced by a paranorm. While considering the paranormed sequence spaces, the topology of the dual space must be carefully chosen since the paranorm on the original space does not necessarily induce a well-behaved topology on the dual space. In such cases, it may be convenient to use the topology of uniform convergence [10] on the closed balls of the original space. In this paper, this topology has been frequently used in establishing the representation formulae for the continuous linear functionals in the dual spaces.

Attempts have been made to obtain general representation theorems for the elements in topological duals of the vector-valued sequence spaces such 
as $[A, P]_{0}\left(\left(E_{k}\right)\right)$ and $[A, P]((E))$, which are introduced and studied by the author in [9]. In fact, all the corresponding results related to the duals of the sequence spaces such as $c_{0}, c, l_{p}$, and $w_{p}$ (see $[4,5,11]$ ) follow as special cases of results established in Section 4. A topological isomorphism is established between $l^{\star}\left(P,\left(E_{k}\right)\right)$ and $l\left(Q,\left(E_{k}^{\star}\right)\right)$, for $1<\inf _{k} p_{k}$. Finally, topological duals of the spaces $l\left(P,\left(E_{k}\right)\right)$, for $0<p_{k} \leq 1$, and $w(P, E)$, for $0<p_{k} \leq 1$, are determined.

2. Definitions and notations. Let $\mathbb{N}$ be the set of natural numbers and $\mathbb{C}$ be the set of complex numbers. Throughout this paper, $E$ and $E_{k}$, for all $k \in \mathbb{N}$, are normed linear spaces. The topological dual of a normed linear space $E$ is denoted as $E^{*}$. It is well known that $E^{*}$ is always a Banach space. The closed unit ball in $E$ (resp., $E_{k}$ ) is denoted as $U$ (resp., $U_{k}$ ). We assume that for the sequence $P=\left(p_{k}\right), p_{k}>0$ for all $k \in \mathbb{N}$, and for the infinite matrix $A=\left(a_{n_{k}}\right)$, $a_{n_{k}} \geq 0$ for all $n, k \in \mathbb{N}$. If $P$ is a bounded sequence, then we write $p_{k}=O$ (1) and $M=\max \left(\sup _{k} p_{k}, 1\right)$. If $\inf _{k} p_{k}>1$, then $Q=\left(q_{k}\right)$ is the sequence for which $1 / p_{k}+1 / q_{k}=1$, for each $k \in \mathbb{N}$. The symbol $z^{k}$ denotes a sequence whose $k$ th term is $z$ and all other terms are 0 . In particular, $e^{k}$ is a sequence with $k$ th term 1 and all other terms 0 . Let $I$ denote the unit matrix, $(\mathbb{C}, 1)$ denote the Cesaro matrix [7], and $D$ denote the upper triangular matrix.

A vector-valued sequence space is a linear space of sequences whose elements are in other linear spaces. The symbol $S(\delta)$ stands for the closed $\delta$-ball centered at the origin $\theta$ of such a sequence space and $g$ denotes the paranorm [7] on a sequence space. Some of the topological properties of the following three vector-valued sequence spaces, which are generated by the infinite ma$\operatorname{trix} A=\left(a_{n_{k}}\right)$, were studied by Maddox $[5,7]$ in the special case when $E_{k}=\mathbb{C}$, for all $k \in \mathbb{N}$ :

(i) $[A, P]((E))=\left\{x=\left(x_{k}\right) \mid x_{k} \in E\right.$, for all $k$ and there exists $l \in E$ such that $\sum_{k} a_{n_{k}}\left\|x_{k}-l\right\|^{p_{k}}$ converges, for all $n \in \mathbb{N}$, and tends to 0 as $n \rightarrow \infty$ \} (in this case we say that $x_{k} \rightarrow l[A, P](E)$ );

(ii) $[A, P]_{0}\left(\left(E_{k}\right)\right)=\left\{x=\left(x_{k}\right) \in \Pi_{k} E_{k} \mid \sum_{k} a_{n_{k}}\left\|x_{k}\right\|^{p_{k}}\right.$ converges, for all $n \in$ $\mathbb{N}$, and tends to 0 as $n \rightarrow \infty\}$;

(iii) $[A, P]_{\infty}\left(\left(E_{k}\right)\right)=\left\{x=\left(x_{k}\right) \in \Pi_{k} E_{k} \mid \sup _{n} \sum_{k} a_{n_{k}}\left\|x_{k}\right\| p_{k}<\infty\right\}$.

If $p_{k}=O(1)$, then the spaces $[A, P]_{0}\left(\left(E_{k}\right)\right),[A, P]((E))$, and $[A, P]_{\infty}\left(\left(E_{k}\right)\right)$ are linear topological spaces [7], the topology being induced by the paranorm

$$
g(x)=\sup _{n}\left[\sum_{k} a_{n_{k}}\left\|x_{k}\right\|^{p_{k}}\right]^{1 / M} .
$$

These three spaces generalize almost all the well-known sequence spaces. For example, if $A=D$, the upper triangular matrix, then $[D, P]_{0}\left(\left(E_{k}\right)\right)=l\left(P,\left(E_{k}\right)\right)$. In particular, if $p_{k}=p$ for all $k$, then we get the sequence space $l\left(p,\left(E_{k}\right)\right)$ (see [5]) and if $E_{k}=\mathbb{C}$ for all $k$, then we get $l(P)$ (see [11]). Moreover, if $E_{k}=\mathbb{C}$ and 
TABLE 2.1

\begin{tabular}{l|ccc}
\hline \multirow{2}{*}{ Matrix } & $\begin{array}{c}\text { Vector-valued sequence } \\
\text { spaces }\end{array}$ & $\begin{array}{c}\text { If } E_{k}=\mathbb{C} \\
\text { for all } k\end{array}$ & $\begin{array}{c}\text { If } E_{k}=\mathbb{C}, p_{k}=1, \\
\text { for all } k\end{array}$ \\
\hline \multirow{3}{*}{$A=I$} & {$[A, P]((E))=c(P, E)$} & $=c(P)$ & $=c$ \\
& {$[A, P]_{0}\left(\left(E_{k}\right)\right)=c_{0}(P, E)$} & $=c_{0}(P)$ & $=c_{0}$ \\
& {$[A, P]_{\infty}\left(\left(E_{k}\right)\right)=l_{\infty}\left(P,\left(E_{k}\right)\right)$} & $=l_{\infty}(P)$ & $=l_{\infty}$ \\
\hline \multirow{3}{*}{$A=(\mathbb{C}, 1)$} & {$[A, P]((E))=w(P, E)$} & $=w(P)$ & $=w$ \\
& {$[A, P]_{0}\left(\left(E_{k}\right)\right)=w_{0}(P, E)$} & $=w_{0}(P)$ & $=w_{0}$ \\
& {$[A, P]_{\infty}\left(\left(E_{k}\right)\right)=w_{\infty}\left(P,\left(E_{k}\right)\right)$} & $=w_{\infty}(P)$ & $=w_{\infty}$ \\
\hline
\end{tabular}

$p_{k}=p$, for all $k$, then we have the special case of $l_{p}$ [7]. Table 2.1 gives a few examples of special cases of $[A, P]_{0}\left(\left(E_{k}\right)\right),[A, P]((E))$, and $[A, P]_{\infty}\left(\left(E_{k}\right)\right)$.

The following spaces are used in establishing some results in Section 4:

$$
\begin{aligned}
M_{1}\left(P,\left(E_{k}^{*}\right)\right) & =\cup_{r>1}\left\{\left(f_{k}\right) \in \Pi_{k} E_{k}^{*} \mid \sum_{k}\left\|f_{k}\right\|^{q_{k}} r^{-q_{k} / p_{k}}<\infty\right\}, \\
M_{2}\left(P,\left(E_{k}^{*}\right)\right) & =\left\{\left(f_{k}\right) \in \Pi_{k} E_{k}^{*} \mid \sum_{r \geq 0} \max _{r} 2^{r / p_{k}}\left\|f_{k}\right\|<\infty\right\}, \\
M_{3}\left(p, E^{*}\right) & =\left\{\left(f_{k}\right) \mid f_{k} \in E^{*}, \forall k, \sum_{r \geq 0} 2^{r / p}\left(\sum_{r}\left\|f_{k}\right\|^{q}\right)^{1 / q}<\infty\right\} .
\end{aligned}
$$

3. Preliminaries. In this section, we introduce the lemmas that are used to prove the main results in Section 4.

LEMMA 3.1. If $p>1, x, y \in \mathbb{C}$, and $1 / p+1 / q=1$, then $|x y| \leq|x|^{p}+|y|^{p}$.

Proof. This follows from the well-known inequality [2, page 17],

$$
a^{p^{\prime}} b^{q^{\prime}} \leq p^{\prime} a+q^{\prime} b
$$

where $p^{\prime}>0, q^{\prime}<1, p^{\prime}+q^{\prime}=1, a \geq 0$, and $b \geq 0$ by putting $p^{\prime}=1 / p, q^{\prime}=1 / q$, $|x|=(1 / p) a^{1 / p}$, and $|y|=(1 / q) b^{1 / q}$.

LEMMA 3.2. Let $p_{k}>1$ and $p_{k}=O(1)$. Then

$$
\left|\sum_{k} a_{k} x_{k}\right| \leq\left[\sum_{k}\left|a_{k}\right|^{a_{k}}+1\right] g(x)
$$

for all $x=\left(x_{k}\right) \in l(p)$ and $a=\left(a_{k}\right) \in l(q)$, whenever $g(x) \leq 1$. 
Proof. By Lemma 3.1, $\left|a_{k} x_{k}\right| / g(x) \leq\left|a_{k}\right|^{a_{k}}+\left|x_{k} / g(x)\right|^{p_{k}}$, and since $g(x)$ $\leq 1$,

$$
\begin{aligned}
\left|\sum_{k} a_{k} x_{k}\right| & \leq g(x) \sum_{k}\left|\frac{a_{k} x_{k}}{g(x)}\right| \\
& \leq g(x) \sum_{k}\left|a_{k}\right|^{q_{k}}+|g(x)|^{1-M} \sum_{k}\left|x_{k}\right|^{p_{k}} \\
& =g(x)\left[\sum_{k}\left|a_{k}\right|^{q_{k}}+1\right] .
\end{aligned}
$$

This proves the lemma.

LEMMA 3.3 (Simons [11, Lemma 4]). Let $0<p_{k} \leq 1$. If $x \in l(p)$ with $\sum_{k}\left|x_{k}\right|^{p_{k}=}$ $\alpha, a \in l_{\infty}(p)$ with $\sup _{k}\left|a_{k}\right|^{p_{k}}=\beta$ and $\beta>1$, then $\sum_{k} a_{k} x_{k}$ converges absolutely and $\left|\sum_{k} a_{k} x_{k}\right| \leq \alpha \beta$.

4. Representation theorems. This section presents the main results established in the paper. Attempts have been made in Theorems 4.1 and 4.2 to obtain some general representation formulae for elements in $[A, P]^{*}(E)$ and $[A, P]_{0}^{*}(E)$, which include many known cases of representation theorems for sequence spaces as special cases.

THEOREM 4.1. Let $A=\left(a_{n k}\right)$ be an infinite matrix with $\lim _{n \rightarrow \infty} a_{n k}=0$ for each $k \in \mathbb{N}$. Then for each $f \in[A, P]^{*}(E)$, there exist uniquely defined functionals $f_{k} \in E^{*}$ for all $k \in \mathbb{N}$ such that

$$
f(x)=f(y)+\sum_{k} f_{k}\left(x_{k}-l\right)
$$

where $x_{k} \rightarrow l[A, P](E)$ and $y=(l, l, l, \ldots)$.

Proof. Since $x_{k} \rightarrow l[A, P](E), \sum_{k} a_{n k}\left\|x_{x}-l\right\|^{p_{k}}$ converges for each $k \in \mathbb{N}$ and tends to 0 as $n \rightarrow \infty$. So it follows that $\lim _{r \rightarrow \infty} \sup _{n \in \mathbb{N}} \sum_{k \geq r} a_{n k}\left\|x_{k}-l\right\| p_{k}=$ 0 . Since $\lim _{n \rightarrow \infty} a_{n k}=0$ for each $k \in \mathbb{N}, z^{k} \in[A, P](E)$ for each $z \in E$. Also $y \in[A, P](E)$ implies that $x-y-\sum_{k=1}^{r}\left(x_{k}-l\right)^{k} \in[A, P](E)$ for each $r \in \mathbb{N}$, where $\left(x_{k}-l\right)^{k}$ denotes the sequence whose $k$ th term is $x_{k}-l$ and all other terms are 0 . Hence,

$$
g\left[(x-y)-\sum_{k=1}^{r}\left(x_{k}-l\right)^{k}\right]=\sup _{n \in \mathbb{N}}\left[\sum_{k \geq r} a_{n k}\left\|x_{k}-l\right\|^{p_{k}}\right]^{1 / M} \longrightarrow 0
$$

as $r \rightarrow \infty$, which implies that

$x-y=\sum_{k=1}^{r}\left(x_{k}-l\right)^{k}, \quad f(x)=f(y)+\sum_{k} f\left(x_{k}-l\right)^{k} \quad$ for each $f \in[A, P]^{*}(E)$. 
To show the continuity of each linear function $f_{k}$ on $E_{k}$, for any fixed $k \in \mathbb{N}$, define $f_{k}: E \rightarrow \mathbb{C}$ by $f_{k}(z)=f\left(z^{k}\right)$ for any $z \in E$. Since $f$ is continuous, for $\epsilon>0$, there exists $\delta>0$ such that $|f(x)|<\epsilon$, whenever $g(x)<\delta$. Now, let $\|z\|<\left(\delta^{M}\right)^{1 / p_{k}} / T_{k}$, where $T_{k}=\sup _{n} a_{n k}$ for each $k$ and $M=\max \left(1, \sup _{k} p_{k}\right)$. It follows that

$$
g\left(z^{k}\right)=\sup _{n}\left(a_{n k}\left\|z_{k}\right\|^{p_{k}}\right)^{1 / M} \leq T_{k}^{1 / M}\left\|z_{k}\right\|^{p_{k} / M}<\delta
$$

so that $\left|f_{k}(z)\right|=\left|f\left(z^{k}\right)\right|<\epsilon$, which proves our assertion. The required representation in (4.1) follows from this. To prove the uniqueness of the functionals $f_{k}$, note that for

$$
f(x)=f(y)+\sum_{k} f_{k}\left(x_{k}-l\right)=f(y)+\sum_{k} g_{k}\left(x_{k}-l\right),
$$

where $x_{k} \rightarrow l[A, P](E)$, we have $y=(0,0,0, \ldots)$ when $x=z^{k}$. Therefore, $f_{k}=g_{k}$ for all $k$.

This completes the proof of Theorem 4.1.

THEOREM 4.2. If $P=\left(p_{k}\right)$ and $A$ as in Theorem 4.1, then each $f \in[A$, $P]_{0}^{*}\left(\left(E_{k}\right)\right)$ has a unique representation of the form

$$
f(x)=\sum_{k} f_{k}\left(x_{k}\right)
$$

where $f_{k} \in E_{k}^{*}$ are uniquely defined functionals determined by $f$ only.

The proof of Theorem 4.2 is omitted since it can be proved following the same line of argument as in Theorem 4.1.

The following results are due to Maddox [8] who calculated the representation formulae for elements in $\mathbb{C}^{*}(E)$ and $\mathbb{C}_{0}^{*}(E)$. Note that $\mathbb{C}(E)$ (resp., $\mathbb{C}_{0}(E)$ ) is a special case of $[A, P](E)$ (resp., $[A, P]_{0}(E)$ ) obtained by substituting $A=I$ and $p_{k}=1$ for all $k \in \mathbb{N}$.

COROLlary 4.3 [8, Proposition 6.14]. The general form of each $f \in \mathbb{C}^{*}(E)$ is $f(x)=f(y)-\sum_{k} f_{k}(l)+\sum_{k} f_{k}\left(x_{k}\right)$ for every $x \in \mathbb{C}(E)$, where $x_{k} \rightarrow l, y=$ $(l, l, l, \ldots), f_{k} \in E^{*}$, and $\sum_{k}\left\|f_{k}\right\|<\infty$.

COROLlary 4.4 [8, Proposition 6.15]. The general form of each $f \in \mathbb{C}_{0}^{*}(E)$ is $f(x)=\sum_{k} f_{k}\left(x_{k}\right)$ for every $x \in \mathbb{C}_{0}(E)$, where $f_{k} \in E^{*}$ and $\sum_{k}\left\|f_{k}\right\|<\infty$.

In the special case, when matrix $A=D$, a topological isomorphism has been established between $[D, P]_{0}^{*}\left(\left(E_{k}\right)\right)$ and $[D, Q]_{0}\left(\left(E_{k}^{*}\right)\right)$ for $1<\inf _{k} p_{k}$ in Theorem 4.5, while in Theorem 4.10, a similar result is established for the topological dual of $[D, P]_{0}\left(\left(E_{k}\right)\right)$ for $0<p_{k} \leq 1$. Note that $[D, P]_{0}\left(\left(E_{k}\right)\right)=$ $l\left(P,\left(E_{k}\right)\right)$. 
Köthe [3] has calculated the topological dual of $l_{p}\left(\left(E_{k}\right)\right)$ in the special case when $p_{k}=p \geq 1$ for all $k$. Later, Maddox [6] calculated $l^{*}(P)$ for $p_{k} \geq 1$ for all $k$. The following theorem generalizes and unifies both these results.

THEOREM 4.5. Let $1<\inf _{k} p_{k}=L$ and $p_{k}=O(1)$. Then for every $f \in l^{*}(p$, $\left(E_{k}\right)$ ), there exist uniquely defined functionals $f_{k} \in E_{k}^{*}$ for each $k$ such that $f(x)=\sum_{k} f_{k}\left(x_{k}\right)$ for all $x \in l\left(P,\left(E_{k}\right)\right)$. Moreover, $\left(f_{k}\right)^{*} \in l\left(Q,\left(E_{k}^{*}\right)\right)$ and the map $T: f \rightarrow\left(f_{k}\right)$ is a topological isomorphism between $l^{*}\left(P,\left(E_{k}\right)\right)$ and $l\left(Q,\left(E_{k}^{*}\right)\right)$.

Proof. By Theorem 4.2, each $f \in l^{*}\left(P,\left(E_{k}\right)\right)$ can be uniquely expressed as $f(x)=\sum_{k} f_{k}\left(x_{k}\right)$ for all $x=\left(x_{k}\right) \in l\left(P,\left(E_{k}\right)\right)$. To prove that $\left(f_{k}\right) \in l\left(Q,\left(E_{k}^{*}\right)\right)$, assume the contrary. So there exists integers $k_{0}<k_{1}<k_{2}<\cdots$ such that

$$
M_{s}=\sum_{k=k_{s-1}}^{k_{s}}\left\|f_{k}\right\|^{q_{k}}>1
$$

for all $s \in \mathbb{N}$. Since $f_{k} \in E_{k}^{*}$, there exists $z_{k} \in U_{k}$ such that

$$
\left\|f_{k}\right\| \leq 2\left|f_{k}\left(z_{k}\right)\right|
$$

Define a sequence $x=\left(x_{k}\right)$ such that

$$
x_{k}= \begin{cases}s^{-1} M_{s}^{-1}\left|f_{k}\left(z_{k}\right)\right|^{q_{k}-1} \operatorname{sgn}\left(f_{k}\left(z_{k}\right)\right) z_{k}, & \text { if } k_{s-1}<k \leq k_{s}, \\ 0, & \text { otherwise }\end{cases}
$$

Then,

$$
\begin{aligned}
\sum_{k=k_{s-1}+1}^{k_{s}}\left\|x_{k}\right\|^{p_{k}} & \leq \sum_{k=k_{s-1}+1}^{k_{s}} s^{-p_{k}} M_{s}^{-p_{k}}\left|f_{k}\left(z_{k}\right)\right|^{q_{k}}\left\|z_{k}\right\|^{p_{k}} \\
& \leq \sum_{k=k_{s-1}+1}^{k_{s}} s^{-L} M_{s}^{-1}\left\|f_{k}\right\|^{q_{k}} \leq s^{-L}
\end{aligned}
$$

for each $s \in \mathbb{N}$. Since $L>1, x \in l\left(P,\left(E_{k}\right)\right)$. But $\sum_{k} f_{k}\left(x_{k}\right)$ diverges since, for each $s \in \mathbb{N}$,

$$
\begin{aligned}
\left|\sum_{k=k_{s-1}+1}^{k_{s}} f_{k}\left(x_{k}\right)\right| & =s^{-1} M_{s}^{-1} \sum_{k=k_{s-1}}^{k_{s}}\left|f_{k}\left(z_{k}\right)\right|^{q_{k}} \\
& \leq 2^{-H} s^{-1} M_{s}^{-1} \sum_{k=k_{s-1}}^{k_{s}}\left\|f_{k}\right\|^{q_{k}}=2^{-H} s^{-1},
\end{aligned}
$$

where $\sup _{k} q_{k}=H$. This leads to a contradiction and therefore $\left(f_{k}\right) \in l\left(Q,\left(E_{k}^{*}\right)\right)$.

Next, define a map $T: l^{*}\left(P,\left(E_{k}\right)\right) \rightarrow l\left(Q,\left(E_{k}^{*}\right)\right)$ by $T(f)=\left(f_{k}\right)$. Using Lemmas 3.1 and 3.2, one can easily show that $T$ is linear and bijective. So it remains to establish the continuity of $T$ and $T^{-1}$ at the zero elements of $l^{*}\left(P,\left(E_{k}\right)\right)$ and 
$l\left(Q,\left(E_{k}^{*}\right)\right)$, respectively. Note that $l^{*}\left(P,\left(E_{k}\right)\right)$ is endowed with the topology of uniform convergence, while $l\left(Q,\left(E_{k}^{*}\right)\right)$ has the usual paranorm topology. To prove the continuity of $T^{-1}$, let $\epsilon>0$ and $y=\left(y_{k}\right) \in S(\delta)$, the closed $\delta$-ball in $l\left(P,\left(E_{k}\right)\right)$. Also let $0<h\left(f_{k}\right)<\min (1, \epsilon)\left(\delta^{M}+1\right)^{-1}$, where $h$ is the paranorm on $l\left(Q,\left(E_{k}^{*}\right)\right)$. It follows from Lemma 3.1 that

$$
\begin{aligned}
|f(y)| & =\left|\sum_{k} f_{k} y_{k}\right|=\sum_{k} \frac{\left\|f_{k}\right\|}{h\left(f_{k}\right)}\left\|y_{k}\right\| h\left(f_{k}\right) \\
& \leq\left\{\sum_{k}\left\|y_{k}\right\|^{p_{k}}+\sum_{k}\left\|f_{k}\right\|^{q_{k}}\left[h\left(f_{k}\right)\right]^{-q_{k}}\right\} h\left(f_{k}\right) \\
& \leq\left\{\sum_{k}\left\|y_{k}\right\|^{p_{k}}+\sum_{k}\left\|f_{k}\right\|^{q_{k}}\left[h\left(f_{k}\right)\right]^{-H}\right\} h\left(f_{k}\right) \\
& \leq\left[g^{M}(y)+1\right] h\left(f_{k}\right)<\epsilon,
\end{aligned}
$$

which implies the continuity of $T^{-1}$.

To prove the continuity of $T$, it is enough to show that $\left|h\left(f_{k}\right)\right|^{H(M-1)}<\delta^{-1 / M}$, whenever $\sup \{|f(x)| \mid x \in S(\delta)\}<1$, for any $\delta^{1-1 / M}>2$. Choose $z_{k} \in U_{k}$ with $\left|f_{k}\left(z_{k}\right)\right| \geq(1 / 2)\left\|f_{k}\right\|$, for each $k \in \mathbb{N}$. Then, for each $n \in \mathbb{N}$, define a sequence $x^{n}=\left(x_{k}^{n}\right)$ by

$$
x_{k}^{n}= \begin{cases}\frac{2^{M / p_{k}} \operatorname{sgn}\left(f_{k}\left(z_{k}\right)\right)\left\|f_{k}\right\|^{q_{k}-1} \delta^{1 / p_{k}} z_{k}}{h\left(f_{k}\right)^{H / p_{k}}\left|h\left(f_{k}\right)\right|}, & \text { if } 1 \leq k \leq n, \\ 0, & \text { if } k>n .\end{cases}
$$

Note that $x^{n} \in S(\delta)$ since $g\left(x^{n}\right) \leq 2 \delta^{1 / M}\left(\left[\sum_{k=1}^{n}\left\|f_{k}\right\|^{q_{k}}\right]^{1 / M} / h\left(f_{k}\right)^{H / M}\right) \leq$ $2 \delta^{1 / M}<\delta$. This implies that $1 \geq\left|f\left(x^{n}\right)\right| \geq \sum_{k=1}^{n} \delta^{1 / p_{k}}\left[h\left(f_{k}\right)\right]^{-H / p_{k}}\left\|f_{k}\right\|^{q_{k}}$ for each $n \geq 1$, whenever $\sup \{|f(x)| \mid x \in S(\delta)\}<1$. Also, since $\delta^{1 / M} \leq \delta^{1 / p_{k}}$,

$$
\begin{aligned}
\delta^{-1 / M} & \geq \sum_{k}\left\|f_{k}\right\|^{q_{k}}\left[h\left(f_{k}\right)\right]^{-H / p_{k}} \\
& \geq \sum_{k}\left\|f_{k}\right\|^{q_{k}}\left[\sum_{r}\left\|f_{r}\right\|^{q r}\right]^{-1 / p_{k}} \\
& \geq \sum_{k}\left\|f_{k}\right\| \geq\left[\sum_{k}\left\|f_{k}\right\|^{q_{k}}\right]^{1 / H},
\end{aligned}
$$

which shows that $h\left(f_{k}\right) \leq 1$. So, $\delta^{-1 / M} \geq\left[h\left(\left(f_{k}\right)\right)\right]^{-H / M}\left[h\left(\left(f_{k}\right)\right)\right]^{H}$, that is, $h\left(f_{k}\right) \leq \delta^{1 / H(-M+1)}$, and therefore, $T$ is continuous.

This completes the proof of Theorem 4.5.

COROLlaRY 4.6 [3]. If $1<p<\infty, 1 / p+1 / q=1$, and $E_{k}$ are Banach spaces, then $l_{p}^{*}\left(\left(E_{k}\right)\right)=l_{q}\left(\left(E_{k}^{*}\right)\right)$ and $l_{1}^{*}\left(\left(E_{k}\right)\right)=l_{\infty}\left(\left(E_{k}^{*}\right)\right)$. 
COROLlary 4.7 [6, Theorem 4]. If $\inf _{k} p_{k}>1$ and $p_{k}=O(1)$, then $l^{*}(P)$ is linearly homeomorphic to the space $l(Q)$.

If the condition $\inf _{k} p_{k}>0$ is removed from the statement of Theorem 4.5, then in that case we have the following partial result which gives a unique representation of each linear functional on $l\left(P,\left(E_{k}\right)\right)$.

THEOREM 4.8. Let $p_{k}>1$ and $\left(p_{k}\right)=O(1)$. Then each $f \in l^{*}\left(P,\left(E_{k}\right)\right)$ can be uniquely expressed as $f(x)=\sum_{k} f_{k}\left(x_{k}\right)$ for all $x=\left(x_{k}\right) \in l\left(P,\left(E_{k}\right)\right)$, where $\left(f_{k}\right) \in M_{1}\left(P,\left(E_{k}^{*}\right)\right)$. Moreover, the map $T: l^{*}\left(P,\left(E_{k}\right)\right) \rightarrow M_{1}\left(P,\left(E_{k}^{*}\right)\right)$, defined by $T(f)=\left(f_{k}\right)$, is a linear homomorphism.

Proof. Following the same line of argument as in Theorem 4.2, it can be shown that each $f \in l^{*}\left(P,\left(E_{k}\right)\right)$ can be uniquely expressed as $f(x)=$ $\sum_{k} f_{k}\left(x_{k}\right)$ for all $x=\left(x_{k}\right) \in l\left(P,\left(E_{k}\right)\right)$, where $f_{k} \in E_{k}^{*}$. To show that $\left(f_{k}\right) \in$ $M_{1}\left(P,\left(E_{k}^{*}\right)\right)$, assume the contrary. So there exists integers $1<k_{0}<k_{1}<k_{2}<\cdots$ such that $M_{s}=\sum_{k=k_{s-1}+1}^{k_{s}}\left\|f_{k}\right\|^{q_{k}} s^{-q_{k} / p_{k}}>1$ for each $s \geq 1$. Also, we can find $z_{k} \in U_{k}$ such that $\left\|f_{k}\right\| \leq 2^{1 / q_{k}}\left|f_{k}\left(z_{k}\right)\right|$, for each $k \geq 1$. Define a sequence $x=\left(x_{k}\right)$ by

$$
x_{k}= \begin{cases}s^{\left(-1+q_{k}\right) / p_{k}} M_{s}^{-1}\left|f_{k}\left(z_{k}\right)\right|^{q_{k}-1} \operatorname{sgn}\left(f_{k}\left(z_{k}\right)\right) z_{k}, & \text { if } k_{s-1} \leq k \leq k_{s}, \\ 0, & \text { otherwise, }\end{cases}
$$

which obviously belongs to $l\left(P,\left(E_{k}\right)\right)$. However, since

$$
\begin{aligned}
\left|\sum_{k=k_{s-1}+1}^{k_{s}} f_{k}\left(x_{k}\right)\right| & =\left.\left|\sum_{k=k_{s-1}+1}^{k_{s}} s^{\left(-1+q_{k}\right) / p_{k}} M_{s}^{-1}\right| f_{k}\left(z_{k}\right)\right|^{q_{k}} \mid \\
& \geq M_{s}^{-1} 2^{-1} s^{-1} \sum_{k=k_{s-1}+1}^{k_{s}}\left\|f_{k}\right\|^{q_{k}} s^{-q_{k} / p_{k}}=\frac{1}{2 s}
\end{aligned}
$$

for each $s \geq 1, \sum_{k} f_{k}\left(x_{k}\right)$ diverges, which leads to a contradiction. So it follows that $\left(f_{k}\right) \in M_{1}\left(P,\left(E_{k}^{*}\right)\right)$.

Clearly, the map $T$ is linear and injective. To show that $T$ is also surjective, let $\left(f_{k}\right) \in M_{1}\left(P,\left(E_{k}^{*}\right)\right)$, that is, there exists an $r \in \mathbb{N}, r>1$, such that $\sum_{k}\left\|f_{k}\right\|^{q_{k}} r^{-q_{k} / p_{k}}<\infty$. It follows from Lemma 3.1 that for any $x \in l\left(P,\left(E_{k}\right)\right)$,

$$
\begin{aligned}
\sum_{k}\left|f_{k}\left(x_{k}\right)\right| & \leq \sum_{k} \frac{\left\|f_{k}\right\|}{r}\left\|x_{k}\right\| r \\
& \leq \sum_{k}\left[\left\|f_{k}\right\|^{q_{k}} r^{-q_{k}}+r^{p_{k}}\left\|x_{k}\right\|^{p_{k}}\right] \\
& \leq r^{-1} \sum_{k}\left\|f_{k}\right\|^{q_{k}} r^{-q_{k} / p_{k}}+r^{M} \sum_{k}\left\|x_{k}\right\|^{p_{k}}<\infty .
\end{aligned}
$$


Let $f(x)=\sum_{k} f_{k}\left(x_{k}\right)$. Observe that

$$
\begin{aligned}
\frac{\left|f_{k}\left(x_{k}\right)\right|}{r g(x)} & \leq \frac{\left\|f_{k}\right\|}{g(x)} \frac{\left\|x_{k}\right\|}{r} \\
& \leq\left(\frac{\left\|f_{k}\right\|}{r}\right)^{q_{k}}+\left(\frac{\left\|x_{k}\right\|}{g(x)}\right)^{p_{k}} \\
& \leq\left\|f_{k}\right\|^{q_{k}} r^{-q_{k}}+\left\|x_{k}\right\|^{p_{k}}[g(x)]^{-M},
\end{aligned}
$$

whenever $g(x) \leq 1$. Therefore,

$$
\begin{aligned}
|f(x)| & \leq r g(x)\left\{\sum_{k}\left\|f_{k}\right\|^{a_{k}} r^{-q_{k}}+\left\|x_{k}\right\|^{p_{k}}[g(x)]^{-M}\right\} \\
& =g(x)\left[\sum_{k}\left\|f_{k}\right\|^{q_{k}} r^{-q_{k} / p_{k}}+\mathbb{N}\right],
\end{aligned}
$$

which establishes the continuity of $f$. This proves Theorem 4.8.

The following result of Maddox [6, Theorem 3] is an immediate consequence of Theorem 4.8 .

COROLlaRY 4.9. If $p_{k}>1$ and $\left(p_{k}\right)=O(1)$, then $l^{*}(P)$ is linearly homomorphic to the space $M_{1}(P)$.

Note that if we impose the extra condition $1<\inf _{k} p_{k}$ in Corollary 4.9, then the space $M_{1}(P)$ coincides with $l(Q)$.

Next, we investigate the topological dual of $l\left(P,\left(E_{k}\right)\right)$ for $0<p_{k} \leq 1$. Köthe [3] calculated $l_{1}^{*}\left(\left(E_{k}\right)\right)$, and later, Simons [11] established a representation theorem for continuous linear functionals on $l(P)$ for $0<p_{k} \leq 1$. The following theorem generalizes these results by establishing a topological isomorphism between $l^{*}\left(P,\left(E_{k}\right)\right)$ and $l_{\infty}\left(P,\left(E_{k}^{*}\right)\right)$ for $0<p_{k} \leq 1$, for all $k \in \mathbb{N}$.

THEOREM 4.10. Let $0<p_{k} \leq 1$ for all $k \in \mathbb{N}$. Then for each $f \in l^{*}\left(P,\left(E_{k}\right)\right)$, there exist uniquely defined functionals $f_{k} \in E_{k}^{*}$, for all $k \in \mathbb{N}$, such that $f(x)=$ $\sum_{k} f_{k}\left(x_{k}\right)$, where $x=\left(x_{k}\right) \in l\left(P,\left(E_{k}\right)\right)$. Moreover, $\left(f_{k}\right) \in l_{\infty}\left(P,\left(E_{k}^{*}\right)\right)$ and the map $T: f \mapsto\left(f_{k}\right)$ is a topological isomorphism between $l^{*}\left(P,\left(E_{k}\right)\right)$ and $l_{\infty}(P$, $\left.\left(E_{k}^{*}\right)\right)$.

Proof. Following the same argument as in Theorem 4.2, one can show that $f(x)=\sum_{k} f_{k}\left(x_{k}\right)$ is the unique representation of $f(x)$, where $f_{k} \in E_{k}^{*}$, for all $k$ and $x=\left(x_{k}\right) \in l\left(P,\left(E_{k}\right)\right)$. To show that $\left(f_{k}\right) \in l_{\infty}\left(P,\left(E_{k}^{*}\right)\right)$, assume the contrary, that is, there exist integers $1<k_{1}<k_{2}<k_{3}<\cdots$ such that $\left\|f_{k_{s}}\right\|^{p_{k_{s}}}>s^{2}$ for each $s \in \mathbb{N}$. For each $k \in \mathbb{N}$, choose $z_{k} \in U_{k}$ such that $\left\|f_{k}\right\| \leq 2\left|f_{k}\left(z_{k}\right)\right|$ and 
define a sequence $x=\left(x_{k}\right)$ by

$$
x_{k}= \begin{cases}\frac{\operatorname{sgn} f_{k}\left(z_{k}\right)}{\left\|f_{k}\right\|} z_{k}, & \text { if } k=k_{s} \text { for each } s \in \mathbb{N}, \\ 0, & \text { otherwise. }\end{cases}
$$

Then

$$
\sum_{k}\left\|x_{k}\right\|^{p_{k}}=\sum_{s} \frac{\left\|z_{k_{s}}\right\|^{p_{k_{s}}}}{\left\|f_{k_{s}}\right\|^{p_{k_{s}}}} \leq \sum_{s} \frac{1}{\left\|f_{k_{s}}\right\|^{p_{k_{s}}}}<\sum_{s} s^{-2}<\infty
$$

so that $x \in l\left(P,\left(E_{k}\right)\right)$. However,

$$
\sum_{k}\left|f_{k}\left(x_{k}\right)\right|=\sum_{s} \frac{\left|f_{k_{s}}\left(z_{k_{s}}\right)\right|}{\| f_{k_{s}} \mid}>\frac{1}{2} \sum_{s} 1,
$$

which contradicts that $\sum_{k} f_{k}\left(x_{k}\right)$ converges. Therefore, $\left(f_{k}\right) \in l_{\infty}\left(P,\left(E_{k}^{*}\right)\right)$.

Next, we show that $T: l^{*}\left(P,\left(E_{k}\right)\right) \rightarrow l_{\infty}\left(P,\left(E_{k}^{*}\right)\right)$, defined by $T(f)=\left(f_{k}\right)$, is a topological isomorphism. Clearly, $T$ is linear and one to one. To show that $T$ is also surjective, let $\left(f_{k}\right) \in l_{\infty}\left(P,\left(E_{k}^{*}\right)\right)$ and $y=\left(y_{k}\right) \in l\left(P,\left(E_{k}\right)\right)$. Then there exists $\alpha>0$ such that $\left\|f_{k}\right\|^{p_{k}} \leq \alpha$ for all $k$, and there exists $k_{0} \in \mathbb{N}$ such that $\left\|y_{k}\right\|^{p_{k}}<1 / \alpha$, for all $k \geq k_{0}$, so that $\left|f_{k}\left(y_{k}\right)\right|^{p_{k}}<1$, for all $k \geq k_{0}$. Then

$$
\sum_{k \geq k_{0}}\left|f_{k}\left(y_{k}\right)\right| \leq \sum_{k \geq k_{0}}\left|f_{k}\left(y_{k}\right)\right|^{p_{k}} \leq \alpha \sum_{k \geq k_{0}}\left\|y_{k}\right\|^{p_{k}}<\infty
$$

which implies that $\sum_{k} f_{k}\left(y_{k}\right)$ converges. Let $f(y)=\sum_{k} f_{k}\left(y_{k}\right)$. The map $f$ is linear and continuous since, by Lemma 3.3, $|f(y)| \leq \alpha g(y)$. So, $f \in l^{*}\left(P,\left(E_{k}\right)\right)$, which implies that $T$ is surjective.

It remains to show the continuity of $T$ and $T^{-1}$ with respect to the topology of uniform convergence on the closed balls of $l\left(P,\left(E_{k}\right)\right)$ and the usual paranorm topology on $l_{\infty}\left(P,\left(E_{k}^{*}\right)\right)$, respectively. To prove the continuity of $T^{-1}$, let $\epsilon>0$ and $x \in S(\beta)$, the closed $\beta$-ball in $l\left(P,\left(E_{k}\right)\right)$. Since $|f(x)| \leq$ $\sum_{k}\left\|f_{k}\right\|\left\|x_{k}\right\| \leq \delta \beta<\epsilon$, whenever $\sup _{k}\left\|f_{k}\right\|^{p_{k}}<\delta$, where $\delta=\min (1, \epsilon) / \beta$, it follows that $T^{-1}$ is continuous.

To prove the continuity of $T$, choose $z_{k} \in U_{k}$ for each $k \geq 1$ such that $(1 / 2)\left\|f_{k}\right\| \leq\left|f_{k}\left(z_{k}\right)\right|$, and for each $n \in \mathbb{N}$, define a sequence $t^{n}=\left(t_{k}^{n}\right)$ by

$$
t_{k}^{n}= \begin{cases}\frac{\operatorname{sgn}\left(f_{k}\left(z_{k}\right)\right) 2^{-1}\left\|f_{k}\right\| \delta^{1 / p_{k}}}{f_{k}\left(z_{k}\right)}, & \text { if } k=n \\ 0, & \text { otherwise }\end{cases}
$$


Then, for each $n \in \mathbb{N}, t^{n} \in s(\delta)$ since

$$
g\left(t^{n}\right)=\sum_{k}\left\|t_{k}^{n}\right\|^{p_{k}} \leq \delta 2^{-p_{n}} \frac{\left\|f_{n}\right\|^{p_{n}}}{\left|f_{n}\left(z_{n}\right)\right|^{p_{n}}}<\delta .
$$

So it follows that $1>\left|f\left(t_{n}\right)\right|=2^{-1} \delta^{1 / p_{n}}\left\|f_{n}\right\|$ for each $n \in \mathbb{N}$, whenever $\sup \{|f(x)| \mid x \in S(\delta)\}<1$. This implies that $T\left(\left(f_{k}\right)\right)<2 \delta^{-1}$, which proves the continuity of $T^{-1}$.

This completes the proof of Theorem 4.10.

The following result is an immediate consequence of Theorem 4.10.

COROLLARY 4.11 [11, Theorem 7]. If $0<p_{k} \leq 1$, then the following statements are equivalent:

(1) the map $\left(x_{k}\right) \mapsto \sum_{k} a_{k} x_{k}$ is a linear functional on $l(P)$;

(2) $\sum_{k} a_{k} x_{k}$ is convergent for all $x=\left(x_{k}\right) \in l(P)$;

(3) $\left(a_{k}\right) \in l_{\infty}(P)$.

Since $l(P)$ admits the Schauder basis [7] $\left(e^{k}\right), f(x)=\sum_{k} f\left(e_{k}\right) x_{k}$, for each $x=\left(x_{k}\right) \in l(P)$. So this result amounts to saying that $l^{*}(P)=l_{\infty}(P)$ when $0<p_{k} \leq 1$, which is a special case of Theorem 4.10 .

The next theorem deals with the special case when $A=(\mathbb{C}, 1)$, the Cesaro matrix. The structure of the topological dual of $[(\mathbb{C}, 1), P](E)=w(P, E)$ is discussed for $0<p_{k} \leq 1$. The calculation of topological dual of $w(P, E)$, for $p_{k}>1$, still remains open.

Borwein [1] determined the structure of the continuous linear functionals on $w_{p}$ for $1 \leq p<\infty$. Later, Maddox [4, 5] investigated $w^{*}(P)$ and $w_{0}^{*}(P)$, for $0<p_{k} \leq 1$.

THEOREM 4.12. Let $0<\inf _{k} p_{k} \leq p_{k} \leq 1$. Then, every $f \in w^{*}(P, E)$ can be uniquely expressed as

$$
f(x)=f(y)+\sum_{k} f_{k}\left(x_{k}\right)-\sum_{k} f_{k}(l),
$$

where $x_{k} \rightarrow l[w(P, E)], y=(l, l, l, \ldots)$, and $f_{k} \in E^{*}$ for all $k$. Moreover, $\left(f_{k}\right) \in$ $M_{2}\left(P, E^{*}\right)$ and the map $f \mapsto\left(f_{k}\right)$ is a linear homomorphism between the spaces $w^{*}(P, E)$ and $M_{2}\left(P, E^{*}\right)$.

Proof. By Theorem 4.1, for each $f \in w^{*}(P, E)$, there exist uniquely defined functionals $f_{k} \in E^{*}$ such that (4.26) holds for each $x=\left(x_{k}\right) \in w(P, E)$, where $\mathrm{x}_{k} \mapsto l[w(P, E)]$. To show that $\left(f_{k}\right) \in M_{2}\left(P, E^{*}\right)$, assume the contrary, that is,

$$
\sum_{r \geq 0} \max _{r} 2^{r / p_{k}}\left\|f_{k}\right\|=\infty
$$


Then, by Dini's theorem [12, page 403], there exists a decreasing null sequence $\left(\epsilon_{k}\right)$ such that $\sum_{r \geq 0} \epsilon_{r} \max _{r} 2^{r / p_{k}}\left\|f_{k}\right\|=\infty$. Choose $z_{k} \in U$ such that $\left\|f_{k}\right\| \leq 2\left|f_{k}\left(z_{k}\right)\right|$ and let $\mathbb{N}_{r}$ be the integer such that $\max _{r} 2^{r / p_{k}}\left\|f_{k}\right\|=$ $2^{r / p_{\mathbb{N}_{r}} \|} f_{\mathbb{N}_{r}} \|$.

Define a sequence $b=\left(b_{k}\right)$ by

$$
b_{k}= \begin{cases}2^{r / p_{\mathbb{N}_{r}}} \epsilon_{\mathbb{N}_{r}} z_{\mathbb{N}_{r}} \operatorname{sgn}\left(f_{\mathbb{N}_{r}}\left(z_{\mathbb{N}_{r}}\right)\right), & \text { if } k=\mathbb{N}_{r}, \text { for each } r \geq 0, \\ 0, & \text { otherwise. }\end{cases}
$$

Since

$$
2^{-r} \sum_{k=2^{r}}^{2^{r+1}}\left\|b_{k}\right\|^{p_{k}}=2^{-r} 2^{r} \epsilon_{\mathbb{N}_{r}}^{p_{\mathbb{N}_{r}}}\left\|z_{\mathbb{N}_{r}}\right\|^{p_{\mathbb{N}_{r}}} \mathbb{N}_{r}^{L} \longrightarrow 0 \quad \text { as } r \longrightarrow \infty,
$$

we can define $f(b)=\sum_{k} f_{k}\left(b_{k}\right)$. On the other hand,

$$
\begin{aligned}
\sum_{k=2^{r}}^{2^{r+1}} f_{k}\left(b_{k}\right) & =\epsilon_{\mathbb{N}_{r}} 2^{r / p_{\mathbb{N}_{r}}}\left|f_{\mathbb{N}_{r}}\left(z_{\mathbb{N}_{r}}\right)\right| \\
& \geq \frac{1}{2} \epsilon_{\mathbb{N}_{r}} 2^{r / p_{\mathbb{N}_{r}}}|| f_{\mathbb{N}_{r}} \|, \quad \text { for each } r \geq 0
\end{aligned}
$$

which implies that

$$
\begin{aligned}
\sum_{r \geq 0} \sum_{k=2^{r}}^{2^{r+1}}\left|f_{k}\left(b_{k}\right)\right| & \geq \sum_{r \geq 0} \frac{1}{2} \epsilon_{\mathbb{N}_{r}} 2^{r / p_{\mathbb{N} r}}\left\|f_{\mathbb{N}_{r}}\right\| \\
& =\frac{1}{2} \sum_{r \geq 0} \epsilon_{\mathbb{N}_{r}} \max _{2^{r} \leq k \leq 2^{r+1}} 2^{r / p_{k}}\left\|f_{k}\right\| \\
& \geq \frac{1}{2} \sum_{r \geq 0} \epsilon_{r} \max _{2^{r} \leq k \leq 2^{r+1}} 2^{r / p_{k}}\left\|f_{k}\right\| .
\end{aligned}
$$

So, it follows from (4.27) that $\sum_{k} f_{k}\left(b_{k}\right)$ diverges, which leads to a contradiction. Therefore, $\left(f_{k}\right) \in M_{2}\left(P, E^{*}\right)$.

Since $\sum_{k} f_{k}\left(x_{k}-l\right)$ converges and

$$
\begin{aligned}
\sum_{k}\left|f_{k}(l)\right| & \leq\|l\| \sum_{r \geq 0} \sum_{k=2^{r}}^{2^{r+1}}\left\|f_{k}\right\| \\
& \leq\|l\| \sum_{r \geq 0} \max _{2^{r} \leq k \leq 2^{r+1}} 2^{r / p_{k}}\left\|f_{k}\right\| \sum_{k=2^{r}}^{2^{r+1}} 2^{r / p_{k}} \\
& \leq\|l\| \sum_{r \geq 0} \max _{2^{r} \leq k \leq 2^{r+1}} 2^{r / p_{k}}\left\|f_{k}\right\|<\infty,
\end{aligned}
$$

for each $x=\left(x_{k}\right) \rightarrow l[w(P, E)], \sum_{k} f_{k}\left(x_{k}\right)$ converges. This shows that the representation in (4.26) is valid. 
Next, define the map $T: w^{*}(P, E) \rightarrow M_{2}\left(P, E^{*}\right)$ by $T(f)=\left(f_{k}\right)$. Clearly, $T$ is linear and injective. To show that $T$ is continuous, define a map $t: w(P, E) \rightarrow E$ by $t(x)=l$, where $x=\left(x_{k}\right) \rightarrow l[w(P, E)]$. Clearly, $t$ is well defined and linear. Next, let $s=\min \left(\|l\|,\|l\|^{p_{k}}\right)$. So, for $\epsilon>0$,

$$
s \leq 2^{-r} \sum_{k=2^{r}}^{2^{r+1}}\|l\|^{p_{k}} \leq 2^{-r} \sum_{k=2^{r}}^{2^{r+1}}\left\|l-x_{k}\right\|^{p_{k}}+2^{-r} \sum_{k=2^{r}}^{2^{r+1}}\left\|x_{k}\right\|^{p_{k}} \leq g(x)+\epsilon,
$$

which implies that $\|t(x)\|=\|l\| \leq(1 / 2)\left[g(x)+g(x)^{1 / L}\right]$ and therefore $t$ is continuous. Then define two maps $h_{1}$ and $h_{2}$ on $w(P, E)$ by $h_{1}(x)=\sum_{k} f_{k}(l)$ and $h_{2}(x)=\sum_{k} f_{k}\left(x_{k}\right)$, respectively. The map $h_{1}$ is continuous since

$$
\begin{aligned}
\left|h_{1}(x)\right| & =\|l\| \sum_{k}\left\|f_{k}\right\|=\|l\| \sum_{r \geq 0} \max _{2^{r} \leq k \leq 2^{r+1}} 2^{r / p_{k}}\left\|f_{k}\right\| \\
& \leq \frac{\beta}{2}\left[g(x)+g(x)^{1 / L}\right],
\end{aligned}
$$

where $\beta=\sum_{r \geq 0} \max _{2^{r} \leq k \leq 2^{r+1}} 2^{r / p_{k}}\left\|f_{k}\right\|$. To prove the continuity of $h_{2}$, let $\alpha=$ $\max (1, g(x))$. Then since

$$
\sum_{k=2^{r}}^{2^{r+1}}\left(\frac{\left\|x_{k}\right\|}{2^{r / p_{k}} \alpha^{1 / l}}\right)^{p_{k}} \leq \frac{g(x)}{\alpha^{1 / l}} \leq \frac{g(x)}{\alpha} \leq 1
$$

it follows that

$$
\begin{aligned}
\sum_{k=2^{r}}^{2^{r+1}}\left|f_{k}\left(x_{k}\right)\right| & \leq \alpha^{1 / L} \max _{2^{r} \leq k \leq 2^{r+1}} 2^{r / p_{k}}\left\|f_{k}\right\| \sum_{k=2^{r}}^{2^{r+1}}\left\|x_{k}\right\| 2^{-r / p_{k}} \alpha^{1 / l} \\
& <\alpha^{1 / l} \max _{2^{r} \leq k \leq 2^{r+1}} 2^{r / p_{k}}\left\|f_{k}\right\| \frac{g(x)}{\alpha}
\end{aligned}
$$

and therefore

$$
\begin{aligned}
\left|h_{2}(x)\right| & \leq \sum_{r \geq 0} \sum_{k=2^{r}}^{2^{r+1}}\left|f_{k}\left(x_{k}\right)\right| \\
& \leq \alpha^{-1+1+/ L}\left[\sum_{r \geq 0} \max _{2^{r} \leq k \leq 2^{r+1}} 2^{r / p_{k}}|| f_{k}||\right] g(x) \\
& \leq \beta \alpha^{-1+1+/ L} g(x),
\end{aligned}
$$

which shows that $h_{2}$ is continuous. Hence $f$ is continuous.

This completes the proof of Theorem 4.12. 
The following two results are special cases of Theorem 4.12.

COROLlaRY 4.13 [4, Theorem 6]. Let $0<\inf _{k} p_{k} \leq p_{k} \leq 1$. Then, for arbitrary $\alpha, a=\left(a_{k}\right) \in M_{2}(P)$, and $x=\left(x_{k}\right) \in w(P)$ with $x_{k} \rightarrow \ell[w(P)], f(x)=$ $\ell \alpha+\sum_{k} a_{k} x_{k}$ defines an element of $w^{*}(P)$. Conversely, every element of $w^{*}(P)$ can be represented in this form.

COROLlaRY 4.14 [4, Theorem 7]. If $0<p_{k} \leq 1$, for all $k$, then $f(x)=$ $\sum_{k} a_{k} x_{k}$ defines an element of $w_{0}^{*}(P)$, for each $x=\left(x_{k}\right) \in w_{0}(P)$, where $\left(a_{k}\right) \in$ $M_{2}(P)$. If, in addition, $0<\inf _{k} p_{k}$, then $f(x)=\sum_{k} a_{k} x_{k}$ is a continuous linear functional on $w_{0}(P)$ if and only if $\left(a_{k}\right) \in M_{2}(P)$.

We have not been able to obtain anything more satisfactory than linear homomorphism for $w^{*}(P, E)$, when $p_{k}=p>1$ for all $k$.

THEOREM 4.15. Let $1<p<\infty$. Then, every $f \in w_{p}^{*}(E)$ can be uniquely expressed as

$$
f(x)=f(y)+\sum_{k} f_{k} x_{k}-\sum_{k} f_{k}(l),
$$

where $x_{k} \rightarrow \ell\left[w_{p}(E)\right], y=l, l, l, \ldots$, and $f_{k} \in E^{*}$ for each $k$. Moreover, $\left(f_{k}\right) \in$ $M_{3}\left(p, E^{*}\right)$ and the map $T: f \mapsto\left(f_{k}\right)$ is a linear homomorphism between the spaces $w_{p}^{*}(E)$ and $M_{3}\left(p, E^{*}\right)$.

The proof of the above theorem is analogous to a result given by Maddox [4] for the corresponding special case $w_{p}$.

The topological dual of $w(P, E)$, for $p_{k}>1$, still remains to be determined. The space $[A, P]_{\infty}^{*}\left(\left(E_{k}\right)\right)$ has also been excluded from the current discussion because even in the special case of $l_{\infty}$, the topological dual is a function space, which will be discussed in a subsequent paper. The study of the three spaces $[A, P]_{\infty}\left(\left(E_{k}\right)\right),[A, P]_{0}\left(\left(E_{k}\right)\right)$, and $[A, P]((E))$ using techniques of functional analysis generalizes and unifies many of the existing results on sequence spaces. Much more has to be investigated on the duals of these spaces. Stated otherwise, topological duals of sequence spaces by matrix transformation can be studied with a new approach and insight with the introduction of these three spaces.

\section{REFERENCES}

[1] D. Borwein, On strong and absolute summability, Proc. Glasgow Math. Assoc. 4 (1960), 122-139.

[2] G. H. Hardy, J. E. Littlewood, and G. Polya, Inequalities, Cambridge University Press, Cambridge, 1967.

[3] G. Köthe, Topological Vector Spaces. I, Die Grundlehren der Mathematischen Wissenschaften, vol. 159, Springer-Verlag, New York, 1969.

[4] I. J. Maddox, Spaces of strongly summable sequences, Quart. J. Math. Oxford Ser. (2) 18 (1967), 345-355. 
[5]__ Paranormed sequence spaces generated by infinite matrices, Math. Proc. Cambridge Philos. Soc. 64 (1968), 335-340.

[6] Continuous and Köthe-Toeplitz duals of certain sequence spaces, Math. Proc. Cambridge Philos. Soc. 65 (1969), 431-435.

[7] _ Elements of Functional Analysis, Cambridge University Press, London, 1970.

[8] _ Infinite Matrices of Operators, Lecture Notes in Mathematics, vol. 786, Springer, Berlin, 1980.

[9] N. Rath, Vector-valued sequence spaces generated by infinite matrices, Int. J. Math. Math. Sci. 26 (2001), no. 9, 547-560.

[10] H. H. Schaefer, Topological Vector Spaces, Graduate Texts in Mathematics, vol. 3, Springer-Verlag, New York, 1971.

[11] S. Simons, The sequence spaces $l\left(p_{v}\right)$ and $m\left(p_{v}\right)$, Proc. London Math. Soc. (3) 15 (1965), 422-436.

[12] K. R. Stromberg, Introduction to Classical Real Analysis, International Mathematics Series, Wadsworth, California, 1981.

Nandita Rath: Department of Mathematics and Statistics, University of Western Australia, Nedlands, WA 6907, Australia

E-mail address: rathn@maths . uwa . edu . au 


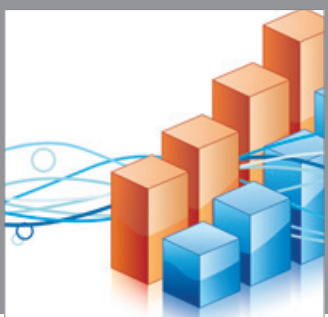

Advances in

Operations Research

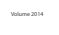

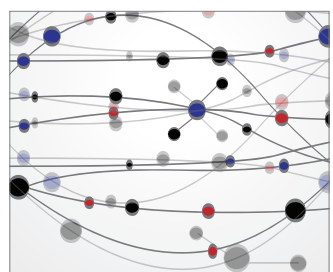

\section{The Scientific} World Journal
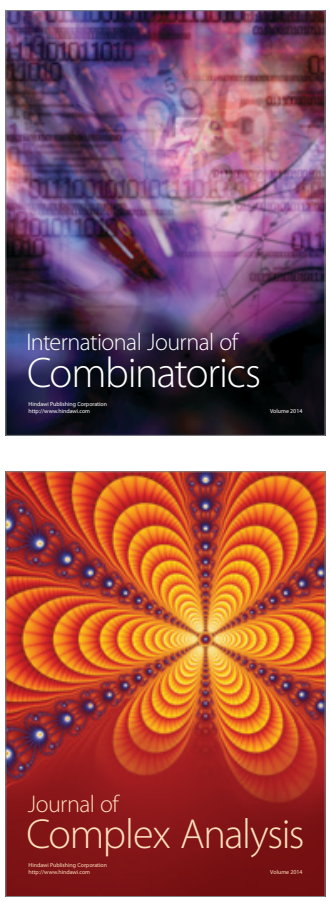

International Journal of

Mathematics and

Mathematical

Sciences
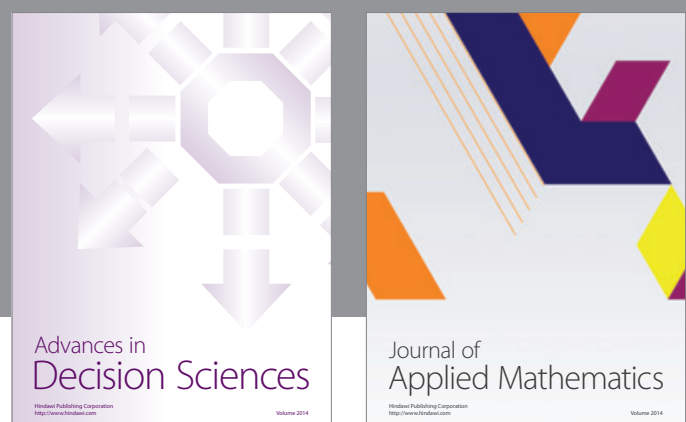

Journal of

Applied Mathematics
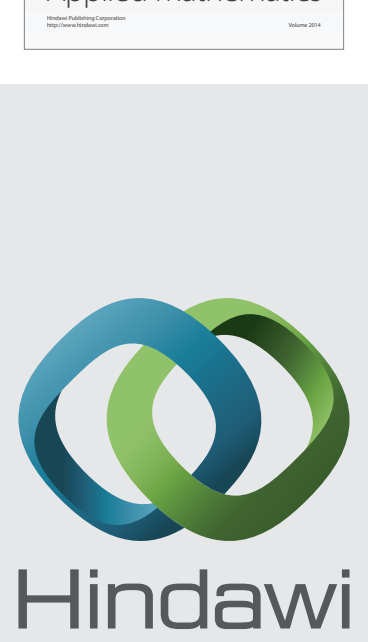

Submit your manuscripts at http://www.hindawi.com
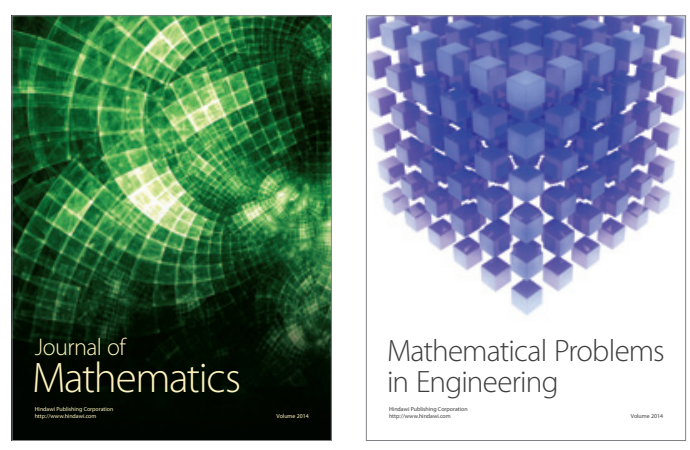

Mathematical Problems in Engineering
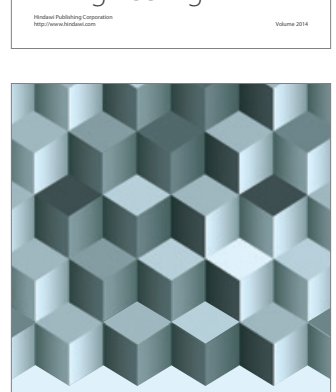

Journal of

Function Spaces
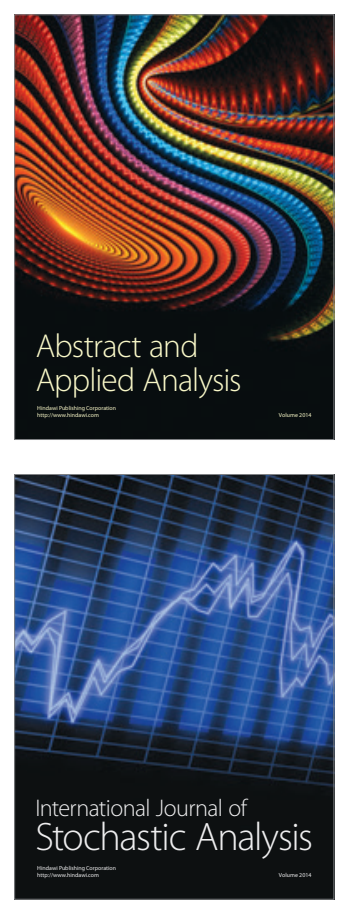

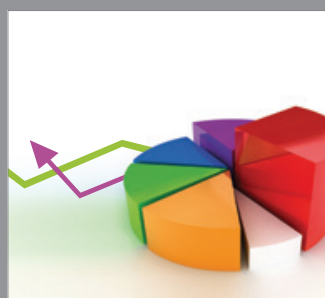

ournal of

Probability and Statistics

Promensencen
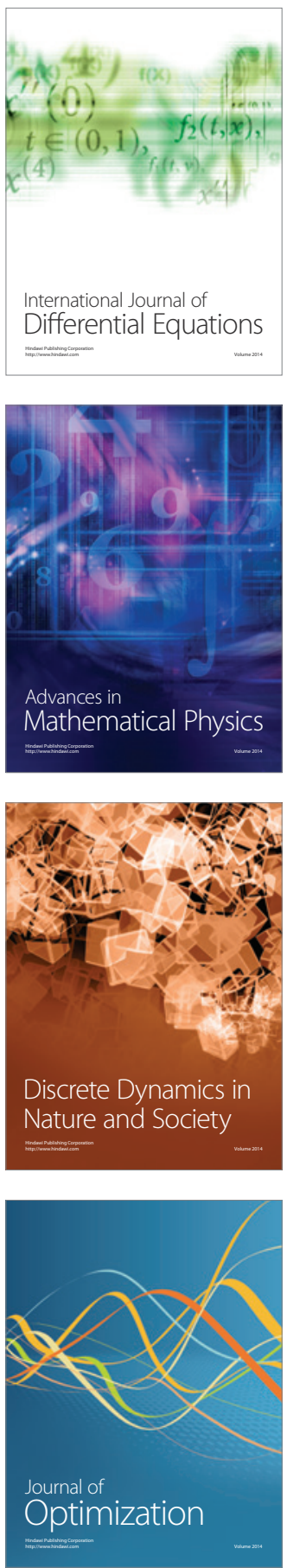